\title{
Prognostic Potential of the Expression of Podoplanin (D2- 40) Within Cells of Squamous Cell Carcinoma of the Larynx and Hypopharynx
}

\author{
Hans-Ullrich Voelker ${ }^{\mathrm{a}, ~}$, Isabelle Hintermeier ${ }^{\mathrm{a}}$, Annette Strehl ${ }^{\mathrm{a}}$, Matthias Scheich ${ }^{\mathrm{b}}$
}

\begin{abstract}
Background: Podoplanin (D2-20) stains immunohistochemically lymphatic vessels, regular mesothelium and tumor cells of different tumors, e.g. malignant mesothelioma or seminoma. In squamous cell carcinoma (SCC), the marker has been described as variously expressed.
\end{abstract}

Methods: This study has investigated the value of the immunohistochemical analysis for the prognostic relevance of the expression in 119 SCCs of the larynx and hypopharynx. The clinical data and documentation of follow-up for at least 5 years were available.

Results: The collective showed the expected distribution of patient age with accentuation of the male sex and a balanced spread of tumor stages including nodal status. The immunohistochemical stain intensity (negative, weak or strong) and the distribution (equal versus focal) were evaluated. In addition, the accentuation of the staining reaction was separately examined at the border of invasion. SCCs with a strong expression of podoplanin were associated with an unfavorable prognosis. A comparison of grouped cases showed a trend emerging with borderline results (negative to weakly positive, $\mathrm{P}=$ 0.51 ; negative to strongly positive, $\mathrm{P}=0.054$; weakly positive to strongly positive, $\mathrm{P}=0.17$ ). The staining at the border of invasion had no statistical effect on overall survival. Multivariate survival statistics however showed that lymphonodal metastasis and a reaction with podoplanin in tumor cells are associated with significant worse prognosis.

Conclusion: In summary, regardless of the exact function of podoplanin in the process of cell migration and tumor progression, an immunohistochemical identification of expression in tumor cells of SCC

Manuscript submitted January 14, 2020, accepted February 18, 2020

aDepartment of Pathology, Leopoldina Krankenhaus GmbH, Gustav-AdolfStr. 8, D-97422 Schweinfurt, Germany

bepartment of Oto-Rhino-Laryngology, Plastic, Aesthetic and Reconstructive Head and Neck Surgery, University Hospital of Wuerzburg, Josef-Schneider-Str. 2, D-97080 Wuerzburg, Germany

${ }^{\mathrm{c} C}$ Corresponding Author: Hans-Ullrich Voelker, Department of Pathology, Leopoldina Hospital, Gustav-Adolf-Str. 8, D-97422 Schweinfurt, Germany. Email: hvoelker@leopoldina.de

doi: https://doi.org/10.14740/wjon1259 of the larynx and hypopharynx can give additional information about the expectable prognosis.

Keywords: Podoplanin; D2-40; Squamous cell carcinoma; Larynx; Hypopharynx

\section{Introduction}

Squamous cell carcinomas (SCCs) are the most common malignant tumors of the larynx and hypopharynx. Some effort has previously been undertaken to find relevant prognostic parameters [1-3]. The monoclonal antibody D2-40 (podoplanin) recognizes a fixation-resistant MR 40’000 O-linked sialoglycoprotein epitope on a transmembrane mucoprotein of lymphatic endothelium. Endothelial cells of blood vessels remain negative in this staining $[4,5]$. Furthermore, D2-40 is expressed in vascular tumors like lymphangioma, Kaposi sarcoma, hemangioendothelioma of the liver as well as in regular mesothelium, malignant mesothelioma, and in some other tumors such as seminoma $[6,7]$. Own previous investigations could illustrate the value of this marker for the evaluation of lymphangioinvasion [6]. During the histological evaluation of staining results for the selective depiction of lymph vessels with D2-40, an additional labeling of tumor cells was observed. As the pattern of expression was seen to be rather variable than constant, we decided to examine the significance of this reaction.

To our knowledge, no previously published study has investigated the value of the immunohistochemical analysis for the prognostic relevance of podoplanin-expression in SCC at this anatomic site. This has already been tested for SCC of other sites. For the project, a cohort of 119 cases of SCC of the larynx or hypopharynx was identified.

\section{Materials and Methods}

A total of 119 archived cases with 70 SCCs of the larynx and 49 SCCs of the hypopharynx were included in this study. The clinical data and documentation of follow-up for at least 5 years were available. Table 1 shows the main case characteristics. All patients included in this study were treated by 
Table 1. Main Characteristics of Cases Included in the Study

\begin{tabular}{lll} 
& Larynx $(\mathbf{n}=\mathbf{7 0})$ & Hypopharynx $(\mathbf{n}=\mathbf{4 9})$ \\
\hline Median age (years) & $62.2(45-85)$ & $57.2(38-75)^{*}$ \\
Male/female & $66: 4$ & $43: 6$ \\
Median tumor size (cm) & $2.5(0.6-5.2)$ & $3.4(1.0-6.5)^{*}$ \\
Stage (pT1/pT2/pT3/pT4) & $11 / 5 / 22 / 32$ & $7 / 10 / 17 / 15$ \\
Grading (G1/G2/G3) & $5 / 43 / 22$ & $1 / 25 / 23$ \\
Median number of investigated lymph nodes & $30.4(4-76)$ & $31.5(5-82)$ \\
pN0/pN+ & $40 / 30(43 \% \mathrm{pN}+)$ & $14 / 35(71 \% \mathrm{pN}+)^{*}$ \\
Median number of nodal metastases in pN+ & $2(1-25)$ & $3(1-17)$ \\
\hline
\end{tabular}

${ }^{*} \mathrm{P}<0.05$.

complete laryngectomy with neck dissection, except laryngeal pT1-tumors (in which nodal metastases were excluded by ultrasound investigation) where the tumor was treated by a local surgery. Apart from these pT1-cases, all others received postoperative radiotherapy.

Well preserved tumor specimens (fixed in 4\% buffered formalin and paraffin embedded; no autolysis; no mechanical or thermal alterations) were prepared for immunohistochemical analysis. One paraffin block per centimeter of tumor diameter was investigated.

For standard staining and immunohistochemistry serially prepared sections were examined. In large tumors, emphasis was placed on the investigation of the lateral and deep tumor margins (border of invasion). The histomorphological diagnosis and grading of each SCC was evaluated by two experienced pathologists. The resected lymph nodes were also evaluated with nodal metastases being classified as $\mathrm{pN}+$ independent of their number and size.

Immunohistochemistry was performed using standard procedures. Briefly, sections $(2-5 \mu \mathrm{m})$ for immunohistochemistry were air-dried overnight (at least $12 \mathrm{~h}$ ), dewaxed and rehydrated in descending concentrations of ethanol before being heated for antigen unmasking in $10 \mathrm{mM}$ citric acid ( $\mathrm{pH}$ 5.5) for $5 \mathrm{~min}$. After rinsing with distilled water, slides were washed in phosphate buffered saline (PBS). For staining, the ADVANCE kit (DAKO, Germany) was used in accordance with the manufacturer's protocol. The antibody for podoplanin (clone D2-40, DAKO, Germany) was used in a dilution of 1:800.

The staining intensity (negative, weak or strong) and the distribution of stained tumor cells (equal versus focal) were evaluated. In addition, the accentuation of the staining reaction at the border of invasion was separately examined. Our chosen method of evaluation follows that of Funayama et al with a three-tiered grading of podoplanin-expression under additional consideration of the staining intensity of immunohistochemical expression. The grading was suggested as follows: grade 1, positive reaction in the cell border of mucosal epithelial cells limited to only the first basal layer (here: staining at the border of invasion); grade 2, positive in the first basal and upper cell layers, but lower than that in the fourth layer (in our study named as focal expression); grade 3: positive from the first basal to the fifth layer and beyond (named as equal expression in our study) [8]. All slides were analyzed microscopically with a standardized magnification $(\times 200)$. For survival analysis, only the overall survival was considered. The disease-free survival was not included.

Data were processed using Microsoft Excel and SPSS. Pearson's Chi-squared test, Student's $t$-test, Mann-Whitney U test or Cox log rank test were performed for descriptive statistic values. Survival analysis was interpreted using KaplanMeier plots.

This retrospective analysis of completely anonymized data was done according to the Declaration of Helsinki in concordance with the general rules of the ethics committee of the University of Wuerzburg (decision 2016010801).

\section{Results}

The collective showed the expected distribution of patient age with accentuation of the male sex and a balanced spread of tumor stages including nodal status (Table 1).

A correlation of D2-40 expression in tumor cells with patient age, tumor size, stage or grading was not detectable. The analysis of the expression in tumor cells was conducted particularly with respect to its importance for development of lymphonodal metastasis. A statistically measurable connection could not be found.

When considering all tumors independent of their location, tumors without expression of D2-40, tumors with weak or strong expression (equally Funayama grade 3, or focally distributed Funayama grade 2) were found. No tumor showed isolated reaction in tumor cells only at the border of invasion (Funayama grade 1). However, there were some cases with Funayama grade 2 or 3 with or without additional expression at the border of invasion (Fig. 1). Histological examples are shown in Figure 2.

Table 2 shows the expression of D2-40 at the border of invasion in relation to the reaction in the remaining tumor cells. A correlation between both could not be found, so that there seem to be two divergent expression patterns which can occur simultaneously or separately. Regarding the accentuation of D2-40-positive cells at the border of invasion, we compared the staining intensity here with the staining intensity in the entire tumor. In positive cases, we found the same intensity in both places. 


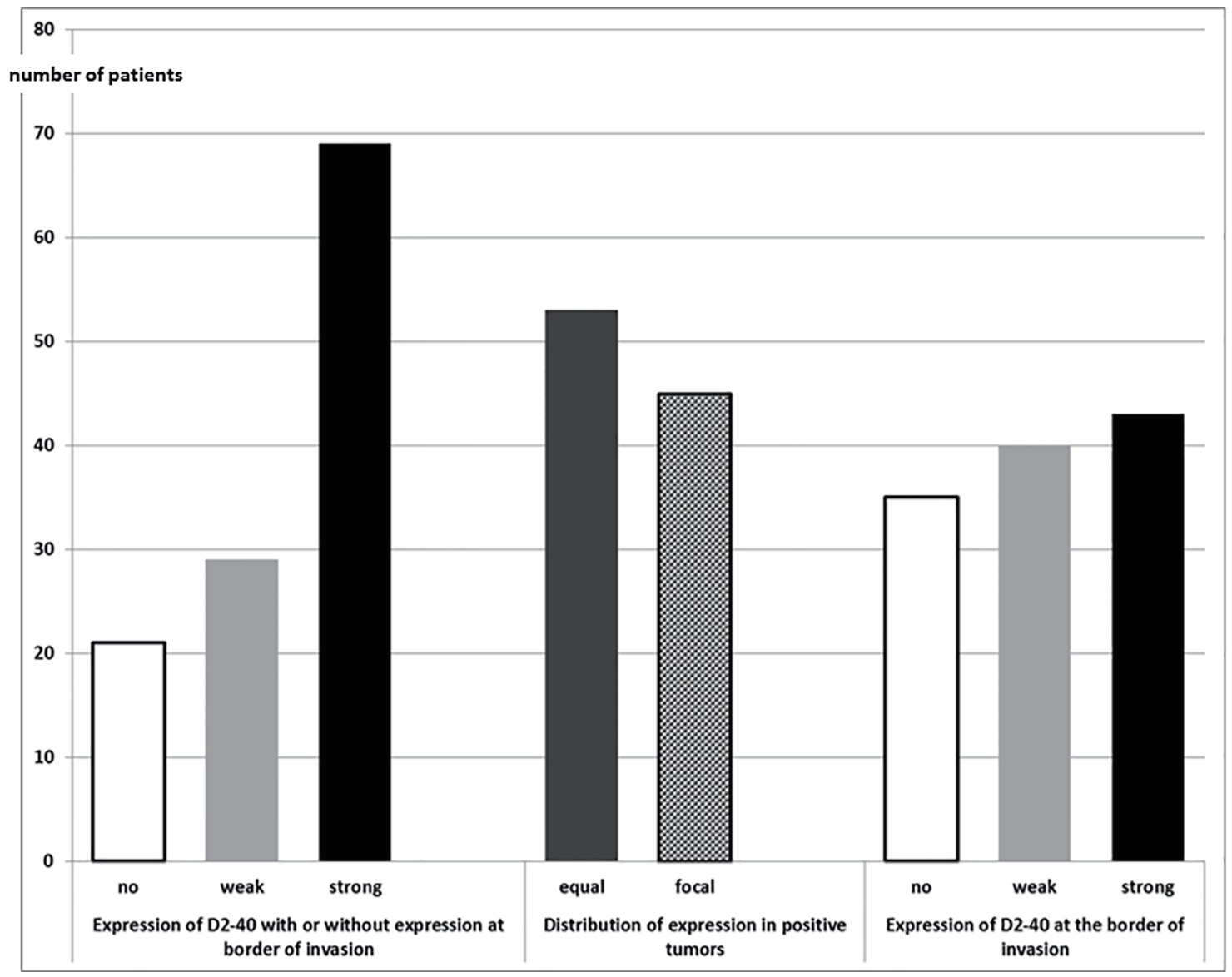

Figure 1. Expression of D2-40 in SCC of larynx and hypopharynx $(n=119)$. SCC: squamous cell carcinoma.

When considering the SCC of the larynx and hypopharynx separately (Table 3 ), we found significantly more carcinomas positive for D2-40 in tumor cells of the larynx $(\mathrm{P}=0.0047)$. The staining intensity was similar for both locations $(\mathrm{P}>0.22)$.

Table 4 shows overall survival with respect to the differing strength of expression of D2-40 (negative, weak or strong expression). Tumors with a strong expression of D2-40 were associated with an unfavorable prognosis. A comparison of grouped cases using the log-rank test was not significant within the groups. However, a trend emerged with borderline results (negative to weakly positive, $\mathrm{P}=0.51$; negative to strongly positive, $\mathrm{P}=0.054$; weakly positive to strongly positive, $\mathrm{P}=$ $0.17)$. Figure 3 shows the survival analysis of negative, weakly and strongly positive SCC.

The distribution of D2-40-positive cells within the tumor (equally Funayama grade 3, or focally distributed Funayama grade 2) showed a surprising result with regard to overall survival. The log-rank test found $\mathrm{P}$ values of 0.38 for all negative compared to all positive tumor cells, of 0.12 for all positive tumor cells compared to focal positivity and of 0.031 for all negative tumors compared to focal positivity.

The staining at the border of invasion had no statistically independent effect on overall survival. Multivariate survival statistics showed that lymphonodal metastasis (details previously given by Voelker et al [6]) and a reaction with D2-40 in tumor cells are associated with an unfavorable prognosis (Table 5).

\section{Discussion}

Podoplanin (D2-40) is a reliable immunohistochemical marker for the depiction of vascular endothelial cells in lymphatic vessels. In addition, there is a wide spectrum of possible reactions in stromal and tumor cells $[6,7]$. Antibody expression in SCC can be disseminated within the tumor cells or focally accentuated in the vicinity of the border of invasion [9-11]. In 2011, Funayama et al suggested a three-tiered grading of immunohistochemical expression [8], which we used as a guideline, with additional consideration of the staining intensity of immunohistochemical expression. The strength of this evaluation scheme was particularly manifested in the differentiation between preneoplastic dysplasia of differing grade and squamous carcinoma in situ. The authors found a positive grade 3 reaction with D2-40 in the tumor in $87 \%$ of the analyzed SCCs and a grade 2 reaction in $10 \%$, whereas only $3 \%$ were exclusively positive at the border of invasion (grade 1). In our study, independently of distribution pattern and intensity of expression, $91 \%$ of laryngeal SCC and $71 \%$ of hypopharyngeal SCC in our study were classified as positive. However, we found few- 


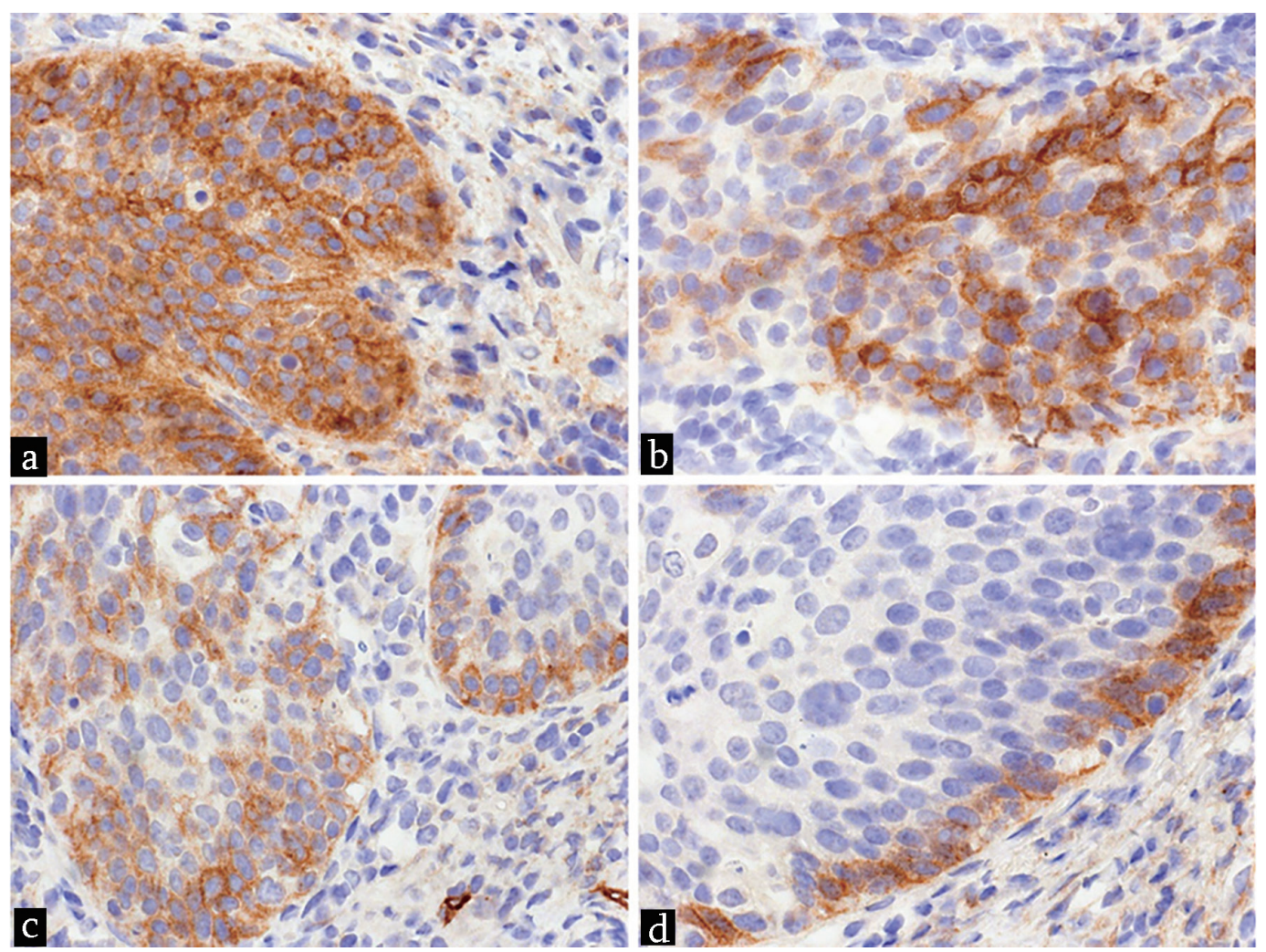

Figure 2. Examples for expression of D2-40 in tumor tissue of SCC. (a) Equally distribution of strong reaction (Funayama grade 3). (b) Focally distribution of strong reaction (Funayama grade 2). (c) Focally distribution of weak reaction. (d) Reaction at the border of invasion within a case with focally weak reaction (not shown in tumor cells nearby the border of invasion within this area) (magnification, $\times 200$ ). SCC: squamous cell carcinoma.

Table 2. Reaction of D2-40 in Tumor Cells With Distribution and Associated Reaction at the Border of Invasion

\begin{tabular}{lllll}
\hline & Reaction in tumor cells & Reaction at border of invasion & $\mathbf{n}$ & $\mathbf{\%}$ \\
\hline \multirow{3}{*}{ Weak expression } & No & No & 21 & 17.6 \\
& Equally & No & 2 & 1.7 \\
& Equally & Positive & 17 & 14.3 \\
& Focally & No & 6 & 5.0 \\
Strong expression & Focally & Positive & 4 & 3.4 \\
& Equally & Positive & 34 & 28.6 \\
& Focally & No & 7 & 5.9 \\
& Focally & Positive & 28 & 23.5 \\
\hline
\end{tabular}

Table 3. Reaction of Tumor Cell in Squamous Cell Carcinoma of Larynx and Hypopharynx With D2-40

\begin{tabular}{|c|c|c|c|c|c|}
\hline & \multicolumn{2}{|c|}{ Larynx } & \multicolumn{2}{|c|}{ Hypopharynx } & \multirow{2}{*}{ P(Chi-square) } \\
\hline & $\mathbf{n}$ & $\%$ & $\mathbf{n}$ & $\%$ & \\
\hline No reaction of tumor cells & 7 & 8.7 & 14 & 28.6 & 0.0047 \\
\hline No reaction at the border of invasion & 16 & 23.2 & 19 & 38.8 & 0.069 \\
\hline Positive reaction at the border of invasion & 53 & 76.8 & 30 & 61.2 & \\
\hline
\end{tabular}

No difference in the intensity of stain $(P>0.22)$. 
Table 4. Survival Depends on Intensity of Stain for D2-40 Within Tumor Cells

\begin{tabular}{|c|c|c|c|c|c|c|c|}
\hline \multirow{2}{*}{ Survival (months) } & \multirow{2}{*}{ All $(n=119)$} & \multicolumn{2}{|c|}{$\operatorname{Negative}(n=21)$} & \multicolumn{2}{|c|}{ Weak expression $(n=29)$} & \multicolumn{2}{|c|}{ Strong expression $(n=69)$} \\
\hline & & $\mathbf{n}$ & $\%$ & $\mathbf{n}$ & $\%$ & n & $\%$ \\
\hline 0 & 119 & 21 & 100 & 29 & 100 & 69 & 100 \\
\hline 12 & 93 & 19 & 95 & 22 & 88 & 52 & 80 \\
\hline 24 & 69 & 15 & 83 & 15 & 66 & 39 & 65 \\
\hline
\end{tabular}

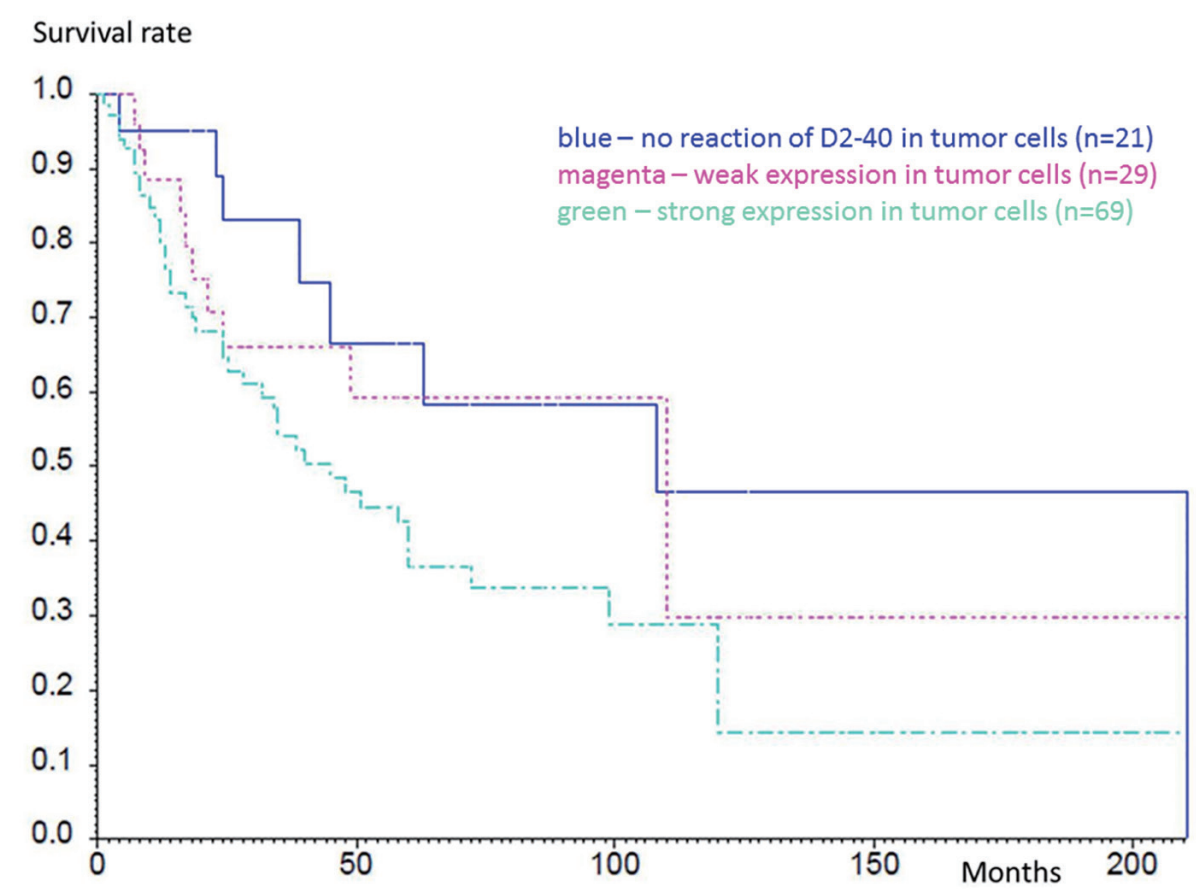

Figure 3. Kaplan-Meier analysis of survival rates within the groups with different expression of D2-40. Blue line shows no reaction of D2-40 in tumor cells $(n=21)$, magenta shows weak expression in tumor cells $(n=29)$ and green shows strong expression in tumor cells $(n=69)$. The differences were not significant.

er SCC with diffuse positivity (equal reaction, grade 3) than Funayama in our collective. An isolated positivity only at the invasion front (grade 1) was not detected.

Functionally podoplanin plays a part in actin remodeling of the cytoskeleton of tumor cells [12]. The results of various studies suggest that podoplanin can assume a biological function in cancer dissemination. The protein potentially promotes communication between neoplastic cells and their surrounding stroma as a basis for locally invasive growth and cell migration into lymphatic vessels. However, the mechanism how tumor cells can enter the lymphatic circulation is not completely understood [13]. Podoplanin is undoubtedly not the only player involved in this complex mechanism and its actual relevance for tumor progression must be further investigated [14]. It is known that podoplanin leads to an increase of the activities of Rho-GTPases, mainly RhoA, whereby different modifications of the cytoskeleton are made possible. An inhibition of RhoA would lead to a reduced motility of tumor cells [15]. Furthermore, podoplanin promotes cell migration of MCF7 cells and HaCaT keratinocytes. The down-regulation of the

Table 5. Multivariate Analysis Shows Lymphonodal Metastases and Positive Reaction for D2-40 as Parameters for Unfavorable Diagnosis

\begin{tabular}{lllll}
\hline & $\mathbf{n}$ & HR & $\mathbf{6 8 \%}$ CI (HR) & P (Chi-square) \\
\hline $\mathrm{pN}+$ & 119 & 2.265 & $1.712(2.996)$ & $0.0035^{* *}$ \\
$\mathrm{D} 2-40$ & 119 & 1.559 & $1.290(1.884)$ & $0.019^{*}$ \\
\hline
\end{tabular}


adhesion molecule E-cadherin subsequently plays a prominent role in tumor spread. Wicki et al could show that an invasion of podoplanin-expressing tumor cells was correlated with an overexpression of matrix metalloproteinases, which facilitated the process of local invasion of tumor cells [10]. De Vicente et al suggested that podoplanin promotes an invasion of tumor cells through increased cell motility formation of filopodialike membrane protrusions. In 92 oral SCCs, they found a diffuse expression of podoplanin in $43 \%$ of well differentiated SCC, with a significantly lower level of expression in moderately differentiated (37\%) and poorly differentiated tumors $(20 \%)$ [12]. An association between expression of podoplanin and the presence of nodal metastases could not be shown; this is consistent with own observations. An interdependency of staining level and tumor grading could also not be observed in our collective. Prasad et al found that $90 \%$ of their collective of oral SCC $(n=30)$ displayed an expression of D2-40 in tumor cells. In contrast to de Vicente et al, they described the highest level of cytoplasmatic expression in poorly differentiated carcinomas (71\%) [16]. The authors discussed the possibility that a stronger expression in tumor tissue could be an indication of a higher number of nodal metastases but could not find this to be statistically significant. In our collective, we could also not demonstrate a correlation between the expression of D2-40 and synchronous nodal metastases. There are however conflicting observations in the relevant literature. Ma et al found a significant connection between expression levels in tumor tissue of 107 esophageal SCC and the development of positive lymph nodes [17], whereas Chuang et al demonstrated a significant correlation between the expression of D2-40 and the occurrence of lymphonodal metastases as well as a more aggressive clinical course in 59 carcinomas of the esophagus [11].

Studies involving tumor tissue from 143 patients with SCC of the cervix uteri demonstrated a significant correlation between the expression of podoplanin in intratumoral stromal fibroblasts (not in the tumor cells!) and lymphonodal metastases [18]. A similar explicit reaction in fibroblasts could not be noted in the own analysis, possibly due to the role played by the antibody clone which was used. Using a multivariate analysis, we could demonstrate the prognostic significance of D2-40 expression as an indication of unfavorable disease progression. This was independent of the level of staining intensity. However, a trend emerged that a stronger expression is associated with a shorter survival time. A larger patient collective could help to clarify this point. Furthermore, the level of staining intensity is possibly conditioned by the level of molecular integrity of the analyzed tumor tissue and the interpretation of these results can be biased by a subjective component. It remains unclear why cases with only focal marker expression show a significantly worse overall survival than cases with evenly distributed expression. One possibility could be differing levels of protein expression at the time of investigation. There is unfortunately no detailed information concerning this in the relevant literature. A factor of less importance in our analysis seems to be that significantly more SCCs of the larynx were found to be positive for D2-40. This distinction from tumors of the hypopharynx could be eliminated in larger collectives as it can be supposed that SCC of neighboring regions should show comparable levels of positivity.

The prognostic relevance of marker expression regardless of other factors has also been described in a collective of 94 cutaneous SCCs, with an expression of podoplanin being associated with an unfavorable prognosis [19]. In oral leukoplakia, an increasingly strong reaction was observed in dysplastic cells correlating with a progression of dysplasia. Therefore, D'souza et al postulated that this is associated with the risk of an aggressive course of disease [20].

The prognostic potential has further been demonstrated in a cohort of $138 \mathrm{SCC}$ of the uterine cervix. In this study, the authors found a shorter recurrence-free survival despite no change of overall prognosis if D2-40 was expressed in the tumor [21]. Because our data do not accurately identify at which point in time a disease progression became manifest, we cannot pursue this observation in our collective.

One consideration that can be found in the relevant literature is whether the expression of podoplanin could be a target for antibody-therapy [21, 22]. Unfortunately, there are no detailed insights on this issue. From our perspective, the available results do not yet allow predictive assessments. Further extensive studies are necessary to prove the extent of the biological importance of podoplanin.

In summary, we can demonstrate that regardless of the exact function of podoplanin in the process of cell migration and tumor progression, a simple and inexpensive immunohistochemical identification of expression of D2-40 in tumor cells of SCC of the larynx and hypopharynx can give additional information about the expectable prognostic development.

HR: hazard ratio.

\section{Acknowledgments}

We thank all technical assistants for their support.

\section{Financial Disclosure}

The authors received no financial support for the research, authorship and/or publication of this article.

\section{Conflict of Interest}

The authors declare no conflict of interest with respect to the research, authorship and/or publication of this article.

\section{Informed Consent}

Permission for studies like that was given by patients within the hospital treatment contract.

\section{Author Contributions}

HUV and MS wrote the manuscript and planned the study. IH 
was involved in whole data collection and statistics. AS and HUV evaluated the immunohistochemically stains.

\section{Data Availability}

The authors declare that data supporting the findings of this study are available within the article.

\section{References}

1. Taguchi T, Nishimura G, Takahashi M, Shiono O, Komatsu M, Sano D, Yabuki KI, et al. Treatment results and prognostic factors for advanced squamous cell carcinoma of the head and neck treated with salvage surgery after concurrent chemoradiotherapy. Int J Clin Oncol. 2016;21(5):869-874.

2. Volker HU, Scheich M, Volter C, Schmidt M, Baier G. [Predictors of nodal metastasising in laryngeal squamous cell carcinomas as decision support for neck dissection: comprehensive analysis of literature]. Laryngorhinootologie. 2008;87(6):392-398.

3. Volker HU, Scheich M, Schmausser B, Kammerer U, Eck M. Overexpression of transketolase TKTL1 is associated with shorter survival in laryngeal squamous cell carcinomas. Eur Arch Otorhinolaryngol. 2007;264(12):14311436.

4. Evangelou E, Kyzas PA, Trikalinos TA. Comparison of the diagnostic accuracy of lymphatic endothelium markers: Bayesian approach. Mod Pathol. 2005;18(11):14901497.

5. Kahn HJ, Marks A. A new monoclonal antibody, D2-40, for detection of lymphatic invasion in primary tumors. Lab Invest. 2002;82(9):1255-1257.

6. Volker HU, Scheich M, Nowack I, Metzger A, Haubitz I, Puppe B, Hagen R, et al. Lymphangiosis carcinomatosa in squamous cell carcinomas of larynx and hypopharynx - value of conventional evaluation and additional immunohistochemical staining of D2-40. World J Surg Oncol. 2009;7:25.

7. Kaiserling E. [Immunohistochemical identification of lymph vessels with D2-40 in diagnostic pathology]. Pathologe. 2004;25(5):362-374.

8. Funayama A, Cheng J, Maruyama S, Yamazaki M, Kobayashi T, Syafriadi M, Kundu S, et al. Enhanced expression of podoplanin in oral carcinomas in situ and squamous cell carcinomas. Pathobiology. 2011;78(3):171-180.

9. Yuan P, Temam S, El-Naggar A, Zhou X, Liu DD, Lee JJ, Mao L. Overexpression of podoplanin in oral cancer and its association with poor clinical outcome. Cancer. 2006;107(3):563-569.

10. Wicki A, Lehembre F, Wick N, Hantusch B, Kerjaschki D, Christofori G. Tumor invasion in the absence of epithelial-mesenchymal transition: podoplanin-mediated remodeling of the actin cytoskeleton. Cancer Cell. 2006;9(4):261-272.

11. Chuang WY, Yeh CJ, Wu YC, Chao YK, Liu YH, Tseng $\mathrm{CK}$, Chang HK, et al. Tumor cell expression of podoplanin correlates with nodal metastasis in esophageal squamous cell carcinoma. Histol Histopathol. 2009;24(8):10211027.

12. de Vicente JC, Santamarta TR, Rodrigo JP, Garcia-Pedrero JM, Allonca E, Blanco-Lorenzo V. Expression of podoplanin in the invasion front of oral squamous cell carcinoma is not prognostic for survival. Virchows Arch. 2015;466(5):549-558.

13. Reis-Filho JS, Schmitt FC. Lymphangiogenesis in tumors: what do we know? Microsc Res Tech. 2003;60(2):171180.

14. Raica M, Cimpean AM, Ribatti D. The role of podoplanin in tumor progression and metastasis. Anticancer Res. 2008;28(5B):2997-3006.

15. Martin-Villar E, Megias D, Castel S, Yurrita MM, Vilaro S, Quintanilla M. Podoplanin binds ERM proteins to activate RhoA and promote epithelial-mesenchymal transition. J Cell Sci. 2006;119(Pt 21):4541-4553.

16. Prasad B, Kashyap B, Babu GS, Kumar GR, Manyam R. Expression of podoplanin in different grades of oral squamous cell carcinoma. Ann Med Health Sci Res. 2015;5(4):299-304.

17. Ma W, Wang K, Yang S, Wang J, Tan B, Bai B, Wang $\mathrm{N}$, et al. Clinicopathology significance of podoplanin immunoreactivity in esophageal squamous cell carcinoma. Int J Clin Exp Pathol. 2014;7(5):2361-2371.

18. Carvalho FM, Zaganelli FL, Almeida BG, Goes JC, Baracat EC, Carvalho JP. Prognostic value of podoplanin expression in intratumoral stroma and neoplastic cells of uterine cervical carcinomas. Clinics (Sao Paulo). 2010;65(12):1279-1283.

19. Canueto J, Cardenoso-Alvarez E, Cosano-Quero A, Santos-Briz A, Fernandez-Lopez E, Perez-Losada J, RomanCurto $\mathrm{C}$. The expression of podoplanin is associated with poor outcome in cutaneous squamous cell carcinoma. J Cutan Pathol. 2017;44(2):144-151.

20. D'Souza B, Nayak R, Kotrashetti VS. Immunohistochemical Expression of Podoplanin in Clinical Variants of Oral Leukoplakia and Its Correlation With Epithelial Dysplasia. Appl Immunohistochem Mol Morphol. 2018;26(2):132-139.

21. Dumoff KL, Chu C, Xu X, Pasha T, Zhang PJ, Acs G. Low D2-40 immunoreactivity correlates with lymphatic invasion and nodal metastasis in early-stage squamous cell carcinoma of the uterine cervix. Mod Pathol. 2005;18(1):97-104.

22. Cirligeriu L, Cimpean AM, Raica M, Doros CI. Dual role of podoplanin in oral cancer development. In Vivo. 2014;28(3):341-347. 